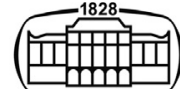

AKADÉMIAI KIADÓ

European Journal of Microbiology and Immunology

10 (2020) 3, 165-171

DOI:

$10.1556 / 1886.2020 .00018$

(C) 2020 The Authors

ORIGINAL RESEARCH PAPER

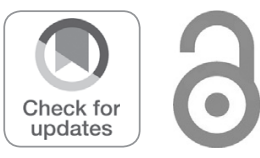

\section{Performance of recombinant proteins in diagnosis and differentiation of canine visceral leishmaniasis infected and vaccinated dogs}

INGRID E. PEREIRA ${ }^{1}$, KYSSIA P. SILVA ${ }^{1}$, LAURA

M. MENEGATI ${ }^{1}$, AIMARA C. PINHEIRO ${ }^{2}$, ELAINE A.

O. ASSUNÇÃO ${ }^{2}$, MARIA DE LOURDES P. ARAÚJO ${ }^{3}$, ELFADIL ABASS ${ }^{4}$, MALCOLM S. DUTHIE ${ }^{5}$, ULRICH STEINHOFF $^{6 \dagger}$ and HENRIQUE C. TEIXEIRA ${ }^{1 * \dagger}$ (])

\author{
${ }^{1}$ Department of Parasitology, Microbiology and Immunology, Institute of Biological Sciences, Federal \\ University of Juiz de Fora, 36036-900, Juiz de Fora, Brazil \\ ${ }^{2}$ Zoonosis Control Center, Municipal Health Secretariat, 35022-280, Governador Valadares, Brazil \\ ${ }^{3}$ Santo Agostinho Veterinary Hospital, 30180-003, Belo Horizonte, Brazil \\ ${ }^{4}$ Department of Clinical Laboratory Science, College of Applied Medical Sciences, Imam \\ Abdulrahman Bin Faisal University, Dammam, Saudi Arabia \\ ${ }^{5}$ HDT Biotech, 98102, Seattle, United States \\ ${ }^{6}$ Institute for Medical Microbiology, Philipps University of Marburg, 35043, Marburg, Germany
}

Received: July 06, 2020 • Accepted: July 13, 2020

Published online: August 27, 2020

\section{ABSTRACT}

Control of canine visceral leishmaniasis (CVL), a major zoonotic disease in Brazil and many other tropical and subtropical countries, remains difficult as an accurate and reliable diagnosis is still missing. In endemic regions, infected dogs are the main parasitic reservoir host of human Visceral leishmaniasis (VL) infection. Vaccination of dogs against Leishmania infection constitutes an important strategy to prevent or to better control CVL, thus, a serological test that can discriminate between antibodies induced by immunization versus infection is highly desirable in order to improve and simplify diagnosis. Here, four recombinant proteins were evaluated for their ability to detect and differentiate between dogs that are infected with Leishmania or have been immunized with the anti-Leishmania vaccine Leish-Tec ${ }^{\mathbb{B}}$. Receiver operating characteristic (ROC) curve analysis of the four Leishmaniaspecific IgG ELISA revealed superior performance of rK28, followed by rKLO8, rK39 and rLb6H. The rK28-based ELISA revealed not only the best accuracy against CVL, but also the lowest cross-reactivity with sera from Leish-Tec ${ }^{\circledR}$ immunized dogs. Our data show that the rK28-based ELISA is highly suitable for CVL screening as it shows high sensitivity with simultaneous low cross-reactivity. Further, the high specificity of the rKLO8 indicates its suitability for the confirmation of CVL diagnosis.

\section{KEYWORDS}

leishmaniasis, canine visceral leishmaniasis, diagnosis, recombinant proteins, accuracy of diagnostic tests, kinesins, rK28, rK39, rKL08, rLb6H

\section{INTRODUCTION}

Visceral leishmaniasis (VL) is a neglected disease that represents a serious public health problem in the more than 70 countries considered endemic [1]. In Brazil, the disease is caused by the protozoan Leishmania infantum, which can be transmitted to man through the bite of the sandfly Lutzomyia longipalpis [2]. Visceral leishmaniasis is a zoonotic disease and among several mammalian hosts, domestic dogs (Canis familiaris) represent the main rural 
and urban reservoir of $L$. infantum. The danger of transmission of VL from dogs to men is high due to their proximity, the susceptibility of dogs to VL infection and the high prevalence of asymptomatic dogs [3]. Canine visceral leishmaniasis $(\mathrm{CVL})$ is a systemic disease which often shows nonspecific clinical signs that are related to the heterogeneity of immune responses induced by Leishmania [4]. Further, CVL may cause severe disease, with high antibody titers and suppression of the cellular immune response or remain asymptomatic through mounting a protective immune response with positive or negative serology for L. infantum [5]. Infected dogs, especially symptomatic animals, are a potential source of $L$. infantum for the vector and thus represent a risk factor for the ongoing transmission to humans [6].

The current gold standard for diagnosing CVL is the positive parasitological examination of aspirates and smears of bone marrow, spleen, liver, lymph node and microscopic analyses of biopsy material from intact or injured skin $[7,8]$. Despite the high specificity, the diagnostic outcome depends on the training and ability of the observer, the parasite load on the infected animal but also the type of immune response developed by the host [9]. While serological tests have been widely used for epidemiological surveys and diagnosis of leishmaniasis [10] one potential disadvantage, especially of those that use crude parasite antigens, is a potential of crossreactivity with other Leishmania species but also trypanosomatids and even phylogenetically distant species that are co-endemic [7].

Several recombinant proteins have been evaluated for their diagnostic potential of human leishmaniasis. For example, the rLb6H protein, identified through screening of a genomic expression library of Leishmania braziliensis, showed good performance in the diagnosis of VL caused by L. infantum and American tegumentary leishmaniasis triggered by the infection of various Leishmania species (L. amazonensis, L. braziliensis, L. guyanensis and L. shawi), while having negligible reactivity to sera from patients with Chagas disease, histoplasmosis, toxoplasmosis, malaria, tuberculosis and paracoccidioidomycosis [11]. Further, the kinesin protein rKLO8, obtained from Leishmania donovani from Sudan, revealed improved sensitivity and specificity especially for detection of specific antibodies in VL patients from East-Africa but also India [12]. Recombinant proteins have also shown promise in the diagnosis of CVL [13], and our recent study showed that the combination of rKLO8 and rK26 proteins increased the sensitivity and specificity of CVL serodiagnosis as compared to monospecific ELISA [14]. Till now, the rK39 and rK28 fusion proteins are routinely used for CVL diagnosis. rK39 has a similar sequence in L. donovani and L. infantum [15] and provides good sensitivity for the diagnosis of symptomatic CVL cases [16]. rK28, a recombinant chimeric protein generated by the fusion of rK39, rK9 and rK26 from L. donovani, is the antigen of choice in an immunochromatographic rapid test that the Brazilian Ministry of Health has recommended for the diagnosis of CVL since 2011. Indeed, the recommendation is that the Dual-Path Platform (DPP; BioManguinhos/Fiocruz, Rio de Janeiro, Brazil) is used in primary screening that is subsequently followed by the ELISABioManguinhos EIE, which uses Leishmania major antigen, as confirmatory test [17].

As vaccination of dogs against Leishmaniasis is an important tool in the fight against Leishmaniasis in Brazil, the differentiation between vaccinated and Leishmaniainfected dogs is crucial for the control of this disease [18]. We thus analyzed the capacity of different recombinant proteins for their diagnostic value to discriminate between dogs that had been tested either positive or negative for CVL or were immunized with the anti-Leishmania amastigotespecific rA2 protein-saponin vaccine Leish-Tec ${ }^{\circledR}$, the only commercially available anti-CVL vaccine in Brazil. Each serum was evaluated using ELISA to assess the reactivity of IgG antibodies against recombinant antigens (rK28, rKLO8, rK39 and rLb6H). Receiver operating characteristic (ROC) curves were then used to evaluate the accuracy of each antigen-specific ELISA, and association between assays were tested by Spearman coefficient correlation.

\section{MATERIAL AND METHODS}

\section{Serum samples}

A total of $88 \mathrm{dog}$ serum samples were obtained from the Zoonosis Control Center serum repository, Governador Valadares, Minas Gerais, Brazil, an area that is endemic for both visceral and tegumentary leishmaniasis. The samples were first screened by the rapid immunochromatographic test TR-DPP ${ }^{\circledR}$ BioManguinhos (DPP) and results were confirmed by the EIE-BioManguinhos (FIOCRUZ, ELISA Kit) as recommended by the Brazilian Ministry of Healthy. The samples were grouped as follows: (i) dogs tested negative for DPP and EIE were considered as endemic control group, EC $(n=44)$. (ii), dogs tested positive for DPP and EIE were grouped as L. infantum seropositive animals, CVL group ( $n=44)$ and (iii), sera from dogs annually vaccinated with the Leish-Tec ${ }^{\circledR}$ vaccine (Ceva Hertape AS, Juatuba, Brazil) but tested negative for leishmaniasis, ehrlichiosis and anaplasmosis were considered as healthy, vaccinated dogs, VAC $(n=44)$. Sera from vaccinated dogs were received from the blood bank of the Santo Agostinho Veterinary Hospital, Belo Horizonte, Minas Gerais. All sera were stored at $-20{ }^{\circ} \mathrm{C}$ until further evaluation.

\section{Recombinant antigens}

The recombinant protein $\mathrm{rKLO} 8$, was cloned and expressed from a Sudanese L. donovani isolate. Briefly, genes were amplified by polymerase chain reaction (PCR) and cloned into the bacterial expression Vector pQ41 (Qiagen $\mathrm{GmbH}$, Germany), containing an N-terminal histidine tag. The protein was over-expressed in Escherichia coli M15 (Qiagen $\mathrm{GmbH}$, Germany) and purified as described previously [12]. Purity and size were checked by gel electrophoresis and 

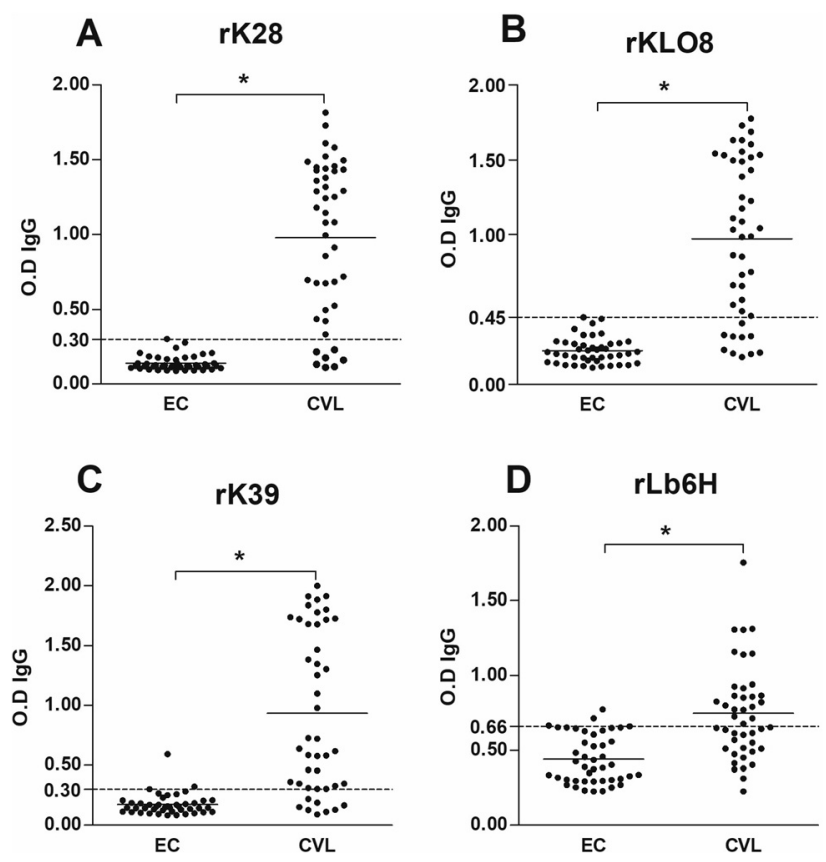

Fig. 1. Antigen-specific antibody responses of dogs with CVL. Levels of IgG antibodies against rK28 (A), rKLO8 (B), rK39 (C) and rLb6H (D) in serum from endemic control dogs (EC; $n=44)$ and dogs with confirmed CVL (CVL; $n=44)$ were measured by ELISA. The cutoff points (dashed bars) were established by the ROC curve. Horizontal bars represent median optical densities determined by

ELISA. Statistical analyses were conducted using the MannWhitney test. ${ }^{*}=P<0.05$

western blotting with anti-His antibodies and sera from VL patients. The recombinant proteins of $L$. donovani, rK28 and rK39 and rLb6H from $L$. braziliensis, were produced at the Infectious Disease Research Institute (IDRI), Seattle, WA, USA. Recombinant antigens were freeze dried and stored at $-80{ }^{\circ} \mathrm{C}$ until evaluation.

\section{Antigen-specific enzyme-linked immunosorbent assay (ELISA)}

Flat-bottom 96-well micro titer plates were coated with 25 $\mathrm{ng} /$ well $(500 \mathrm{ng} / \mathrm{mL})$ of either rK28, rKLO8, rK39 or rLb6H diluted in $0.1 \mathrm{M} \mathrm{NaCO}$ buffer ( $\mathrm{pH}$ 9.6) solution and incubated overnight at $4{ }^{\circ} \mathrm{C}$. Plates were than washed with phosphate buffered saline (PBS) containing 0.05\% Tween-20 (PBS-T) and blocked with blocking solution (2\% BSA in PBS-T) for $1 \mathrm{~h}$ at room temperature. After washing with PBS-T, plates were loaded with serum samples diluted 1:2,000 and incubated at room temperature for $1 \mathrm{~h}$. After washing with PBS-T, $50 \mu \mathrm{L}$ of peroxidase-conjugated rabbit anti-dog IgG (1:5,000 dilution) (Sigma, cod. A6792, St. Louis, MO, USA) were added and incubated at room temperature for $1 \mathrm{~h}$. The plates were then washed again and a substrate solution containing o-phenylenediamine dihydrochloride (OPD) and hydrogen peroxide $\left(\mathrm{H}_{2} \mathrm{O}_{2}\right)$ was used. After color development, the reaction was stopped by the addition of $2 \mathrm{~N} \mathrm{H}_{2} \mathrm{SO}_{4}$ and the color-reaction was read at 492 nm with a Spectramax-190 ELISA reader (Molecular
Devices, Sunnyvale, CA, USA). The results were expressed as absorbance, which corresponds to the mean values of the optical density (OD) of each sample.

\section{Statistical analysis}

Statistical analysis of mean optical densities (ODs) from CVL and EC groups was performed by Mann-Whitney U test. Comparisons of the mean ODs from CVL, EC and VAC groups were performed using Kruskal-Wallis test, followed by Dunn's Multiple Comparison Test (GraphPad Software, San Diego, CA, USA). ROC areas of the tested recombinant proteins were evaluated by the nonparametric Wilcoxon Test, using MEDCALC 14.8.1 (MedCalc Software, Oostende, Belgium). The values of sensitivity and specificity were extracted at the best cutoff point given by each curve. Correlation of the four antigen-specific ELISAs were tested using the Spearman nonparametric method. $P$ values of $<0.05$ was considered statistically significant.

\section{Ethics}

The Ethics Committee on Animal Experimentation of the Federal University of Juiz de Fora approved the study protocol (No. 009/2017).

\section{RESULTS}

\section{Detection of IgG antibodies against recombinant Leishmania antigens}

First, we analyzed sera from dogs with CVL and comparable controls from the same endemic area (EC group) by testing each sample for the presence of IgG antibodies reacting with the recombinant antigens ( $\mathrm{rKLO} 8, \mathrm{rK} 28, \mathrm{rK} 39$ and $\mathrm{rLb} 6 \mathrm{H}$ ) in ELISA. Cut-off values for positive responders were determined by receiver operating characteristic (ROC) analysis. Fig. 1 shows that IgG levels against each of the antigens tested were significantly increased in the CVL group as compared to the EC group. While the magnitude of the antigen-specific reactivity was quite variable, particularly when sera were tested against the rLb6H protein (Fig. 1D), the mean ODs were similar for $\operatorname{rKLO} 8(0.97 \pm 0.52)$, rK28 $(0.97 \pm 0.52)$ and $\mathrm{rK} 39(0.93 \pm 0.67)$, with a lower value when $\mathrm{rLb} 6 \mathrm{H}$ antigen was used $(0.75 \pm 0.31)$.

\section{Comparative evaluation of four recombinant proteins for diagnosis of CVL}

ROC curve analysis was performed to evaluate the accuracy of each antigen-specific ELISA for the diagnosis of CVL (Fig. 2). Represented by the values of the area under the curve (AUC), we observed a similar performance of rK28 $(0.944 ; 95 \% \mathrm{CI}=0.874-0.982)$ and $\mathrm{rKLO} 8(0.926 ; 95 \%$ $\mathrm{CI}=0.850-0.971)$. Although the AUC value of rK39 (0.890; $95 \% \mathrm{CI}=0.806-0.947)$ was statistically not different from rKLO8, it was lower than that of rK28 $(P<0.05)$. The AUC value for $\mathrm{rLb} 6 \mathrm{H}$ was $0.813(95 \% \mathrm{CI}=0.716-0.888)$ and thus significantly lower than those observed for rKLO8 and rK28 


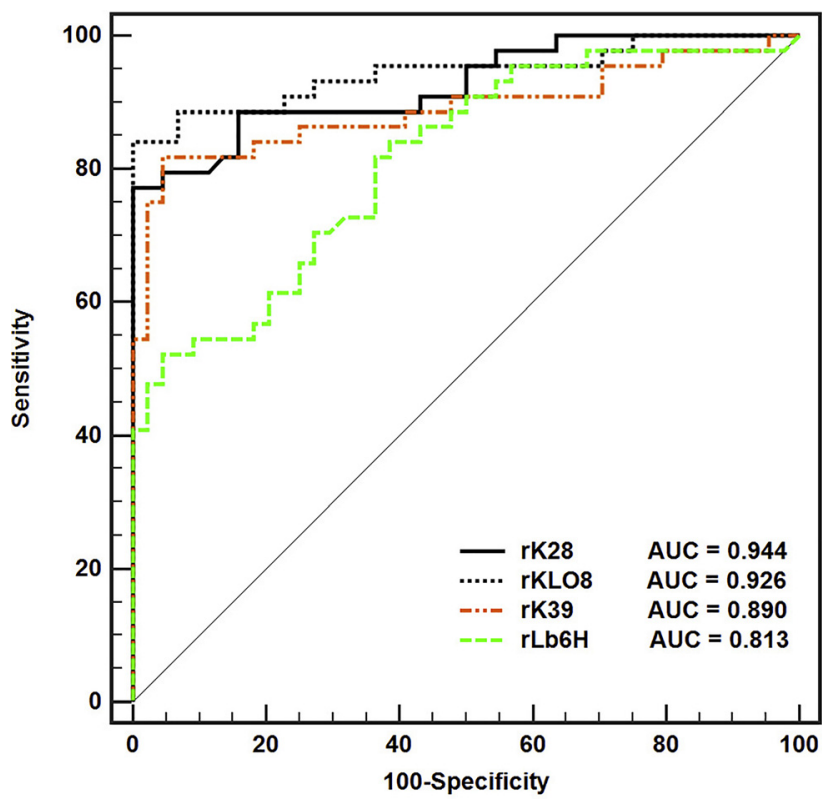

Fig. 2. Diagnostic performance of antigen-specific ELISA. ROC curve analysis of ELISA data were performed, comparing the areas under the curve (AUC) for the rKLO8 $\times$ rK28 $(P=0.4287)$, $\mathrm{rKLO} 8 \times \mathrm{rK} 39(P=0.1246), \mathrm{rK} 28 \times \mathrm{rK} 39(P=0.0258), \mathrm{rKLO} 8 \times$ rLb6H $(P=0.0011), \mathrm{rK} 28 \times \operatorname{rLb} 6 \mathrm{H}(P=0.0013)$ and $\mathrm{rK} 39 \times$ $\mathrm{rLb6H}(P=0.0589)$

(both $P<0.05$; Fig. 2). Spearman rank order correlations revealed a strong linear correlation between the ELISA results with rK28, rKLO8 and rK39, while all of them revealed poor correlation with the rLb6H (Supporting information Fig.).

We further determined the sensitivity and specificity for each recombinant protein by ROC curve analysis. Experiments were performed on separate occasions to demonstrate assay robustness (Table 1). In particular, rK28 and rKLO8 demonstrated high specificity, indicating their usefulness in the exclusion of truly negative dogs from false positive CVL diagnosis. In terms of sensitivity, rK28 and rK39 antigens were the most sensitive with $84 \%$ and $82 \%$, respectively,

Table 1. Performance characteristics of each antigen-specific ELISA

\begin{tabular}{lcccc}
\hline ELISA & Exp. & $\begin{array}{c}\text { Cut-off } \\
\left(A_{450}\right)\end{array}$ & $\begin{array}{c}\text { Sensitivity \% } \\
{[95 \% \mathrm{CI}]}\end{array}$ & $\begin{array}{c}\text { Specificity \% } \\
{[95 \% \mathrm{CI}]}\end{array}$ \\
\hline rK28 & 1 & 0.30 & $84[69.9-93.4]$ & $100[92-100]$ \\
& 2 & 0.23 & $84[69.9-93.4]$ & $95[84.5-99.4]$ \\
rKLO8 & 1 & 0.45 & $77[62.2-88.5]$ & $100[92-100]$ \\
& 2 & 0.41 & $75[59.7-86.8]$ & $93[81.3-98.6]$ \\
rK39 & 1 & 0.30 & $82[67.3-91.8]$ & $95[84.5-99.4]$ \\
& 2 & 0.20 & $82[67.3-91.8]$ & $86[72.6-94.8]$ \\
rLb6H & 1 & 0.66 & $52[36.7-67.5]$ & $95[84.5-99.4]$ \\
& 2 & 0.35 & $57[41.0-71.7]$ & $95[84.5-99.4]$ \\
\hline
\end{tabular}

The cut-off, sensitivity and specificity were determined by receiver operating characteristic (ROC) curve. Data represent values obtained in two independent experiments with sera from negative controls (DPP-/EIE-; $n=44)$ and from CVL affected dogs $(\mathrm{DPP}+/ \mathrm{EIE}+; n=44)$.
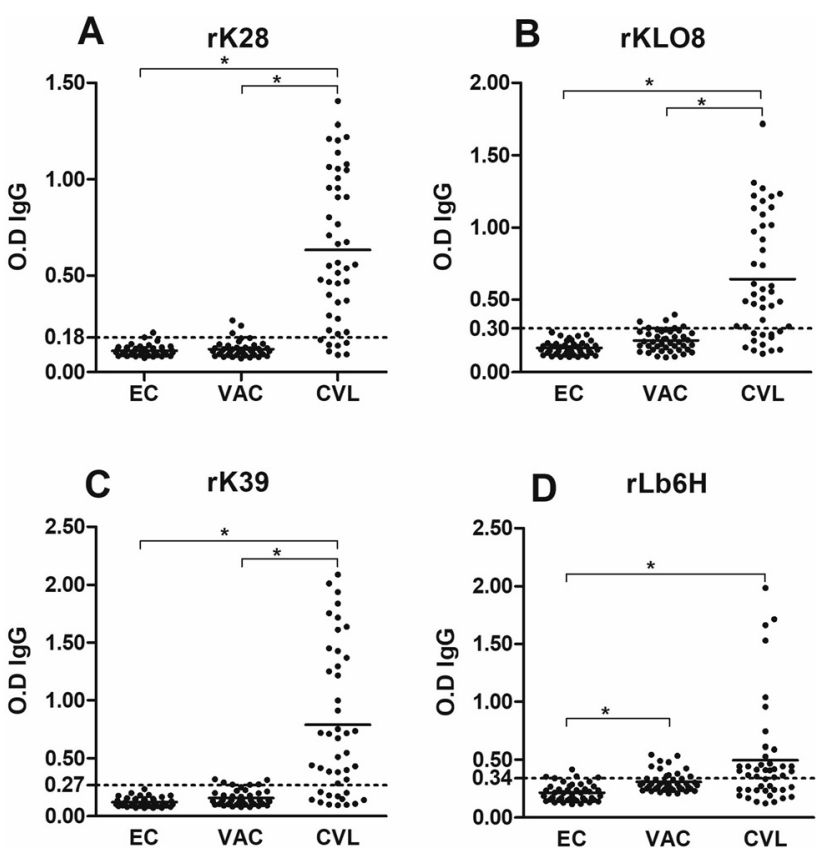

Fig. 3. Antigen-specific antibody responses in vaccinated and unvaccinated dogs. Levels of IgG antibodies against rK28 (A), rKLO8 (B), rK39 (C) and rLb6H (D) in serum from endemic control dogs (EC; $n=44)$, dogs immunized with Leish-Tec ${ }^{\mathbb{B}}$ (VAC; $n=44$ ), and dogs with confirmed CVL (CVL; $n=44$ ) were measured by ELISA. The cut-off point (dashed line) was established by the ROC curve while the horizontal bars represent median OD determined by ELISA. ${ }^{*}=P<0.05$

indicating their suitability for diagnosis of CVL. This was followed by rKLO8 (75-77\%), while rLb6H antigen showed a marked lower sensitivity (52 and 57\%). The highest sensitivity and specificity were given by the rK28-based ELISA. Thus, our data support the superior role of $\mathrm{rK} 28$ in screening for and detection of CVL.

\section{Ability of recombinant proteins to discriminate between infection and vaccination}

Vaccination of dogs is an important control measure against CVL infection. However, serological tests can be problematic as vaccinated animals may be mistaken for infection. We thus tested our recombinant antigens on reacting with antibodies that have been generated after immunization with Leish-Tec ${ }^{\circledR}$ vaccine. While sera obtained from dogs vaccinated with Leish-Tec ${ }^{\circledR}$ were similar to EC in their response to rK28, rK39 or rKLO8, the rLb6H antigen showed significantly increased reactivity with sera from vaccinated animals as compared to the EC group (Fig. 3). Although responses were lower relative to CVL samples, $25 \%$ of the sera from vaccinated dogs $(11 / 44)$ had detectable antibodies against the rLb6H antigen. In contrast, the proportion of reactive sera in the VAC group was lower for rKLO8 and rK39 (both 13\%), and was lowest for rK28 (4.5\%). Together, these data indicate that among the recombinant proteins, rK28-based ELISA had the best ability to discriminate diseased dogs (CVL) from both EC and LeishTec $^{\circledR}$ immunized dogs. 


\section{DISCUSSION}

Efficient screening and diagnosis of CVL infection is important as dogs are a reservoir of infection also for human visceral leishmaniasis [3]. To explore the performance of various recombinant antigens in CVL diagnosis, we tested and compared four recombinant proteins for their ability to detect and differentiate CVL from endemic negative controls and Leish-Tec ${ }^{\circledR}$ vaccinated animals. Our data revealed that sera from dogs with CVL show elevated levels of IgG against all recombinant proteins tested, i.e. rK28, rKLO8, rK39 and rLb6H. While rK28, rKLO8 and rK39 showed a similar reactivity in ELISA, we further distinguished the performance of each protein by ROC curve analysis which revealed superior performance of rK28 ELISA, followed in order by rKLO8, rK39 and, finally, rLb6H. In addition, rK28 stood out, as it not only differentiates between infected and vaccinated dogs but also revealed best diagnostic accuracy.

Although each CVL case in our study was screened before by their positive responses in both DPP and EIEBioManguinhos tests as recommended by the Brazilian Ministry of Health, we observed in our ELISA based test format a broad range of reactivity for each antigen tested. This variability might be due to individual immune responses of the animals, differences in parasite load or the phase of disease, each of which is known to influence the diagnosis of CVL [19]. In addition, the type of test (e.g., DPP and ELISA) and the antigen(s) used for the test have been shown to influence the outcome $[5,20]$.

In particular, the high incidence of false negative but also false positive responses in CVL have been attributed to the use of crude, unfractionated parasite antigens. False positive results can lead to the unnecessary euthanasia of dogs while false negative results bear the risk for continued transmission of infection and persistence of disease [21]. In contrast, using recombinant proteins for diagnosis offers the opportunity to rationally select specific or even autochthonous antigen of a distinct parasite strain with the goal to enhance the specificity and sensitivity with equally increased reproducibility and reduced production costs [10, 22]. With regard to the rK39 antigen, it shares identical amino acid sequences between $L$. donovani and $L$. infantum similar to the fusion antigen $\mathrm{rK} 28$ (K39, K26 and K9) [23, 24]. In contrast, rKLO8 is based on a kinesin sequence of a $L$. donovani isolate from Sudan that exhibits $93 \%$ identity with the kinesin protein of L. infantum [12]. The combination of the rKLO8 and rK26 antigens resulted in enhanced sensitivity and specificity for CVL [14].

The present study demonstrated that rK28 gives the strongest IgG response in dogs that have been positively tested for CVL, with a sensitivity of $84 \%$, followed by rK39 and rKLO8 proteins. The high sensitivity and specificity of the ELISA employing rK28, rKLO8 and rK39 are consistent with other studies using the same antigen [14, 24-26]. Overall, our analyses revealed that the positive and negative predictive values (PPV and NPV, respectively) of rK28 and
rKLO8 were higher than those observed with the rK39-based ELISA (data not shown). The superiority of the rK28 antigen that provides high sensitivity and specificity especially in the diagnosis of CVL symptomatic dogs agrees with previous findings by Grimaldi et al. [5].

While vaccination of dogs represents an important medical intervention to break the chain of transmission between dogs and from dogs to men, a quick and reliable distinction between vaccinated and infected animals still remains an important issue. Although vaccination with Leish-Tec ${ }^{\circledR}$ does not prevent infection, it reduces the parasite load and thus limits the transmission potential of infected dogs [17]. Our present study further shows that few sera from vaccinated, but healthy dogs, exhibited substantial cross-reactivity with $\mathrm{rLb} 6 \mathrm{H}$-antigen, indicating the necessity to carefully evaluate every test-antigen for crossreactivities and false positive results. A study by Fernandes et al. [21] suggests that this is even worse if a crude antigen preparation of $L$. infantum are used for diagnostic purposes. Nevertheless, our data are consistent with studies in which humoral responses induced by Leish-Tec ${ }^{\circledR}$ vaccination can be detected by rA2-ELISA, but do not interfere with the DPP-determined K28-specific antibody reactivity $[27,28]$.

Another important issue for accurate diagnosis of CVL is the potential cross-reactivity with coinfections of related Leishmania species or even different pathogens. While VL in America is typically caused by $L$. infantum, the tegumentary form is caused mainly by L. braziliensis and L. amazonensis. Given the high genetic homology between these species (69$90 \%)$ there is a high potential of cross-reactivity for serodiagnosis of CVL which was previously shown in dogs infected with L. braziliensis and L. amazonensis [5, 29-31]. However, the high sensitivity and specificity of the recombinant antigens used in our study implies that co-infection with other Leishmania species or pathogens phylogenetically close to Leishmania, i.e. Trypanosoma cruzi or Ehrlichia canis may not be a major concern.

Taken together, our data demonstrate that rK28, rKLO8 and rK39 antigen-based ELISA provide good accuracy for the diagnosis of CVL and to differentiate between vaccinated and infected dogs. The high sensitivity of rK28 supports its use for CVL screening. The high specificity of rKLO8 makes this antigen suitable to confirm CVL diagnosis. Further studies with a new fusion protein that includes various dominant kinesin epitopes from several Leishmania strains will be used to accelerate and increase the serodiagnosis of CVL and human VL.

Funding sources: This work was supported by Conselho Nacional de Pesquisa e Desenvolvimento Tecnológico (CNPq, Project 31036/2015-5 to HCT) and Fundação de Amparo à Pesquisa do Estado de Minas Gerais (FAPEMIG, Project RED-00313-16 to HCT). IEP received a doctoral fellowship from Coordenação de Aperfeiçoamento de Pessoal de Nível Superior (CAPES, Brazil). The work was also supported by the LOEWE Center DRUID (Projects C4 to US) within the Hessian Excellence Program. 
Authors' contribution: HCT, US and MSD conceived the study; HCT and IEP wrote the initial draft of the manuscript; IEP, KPS and LMM performed the ELISA experiments, ROC curve and Spearman's correlation analysis; ACP, EAOA and MLPA carried out clinical CVL assessment and acquisition of serum samples; MSD, EA, UE performed recombinant proteins preparation. US obtained funding for publication costs. All authors contributed to the analysis and interpretation of the data and had full access to all data in the study and take responsibility for the integrity of the data and the accuracy of the data analysis.

Conflict of interests: The authors certify that there is no conflict of interest.

\section{ACKNOWLEDGMENTS}

We are grateful for the cooperation of the staff of both the Zoonosis Control Center of Governador Valadares and Santo Agostinho Veterinary Hospital of Belo Horizonte.

\section{SUPPLEMENTARY MATERIAL}

Supplementary data to this article can be found online at https://doi.org/10.1556/1886.2020.00018.

\section{REFERENCES}

1. World Health Organization. Leishmaniasis, epidemiology situation in 2017. Available from: https://www.who.int/leishmaniasis/ burden/en/.

2. Maroli M, Feliciangeli MD, Bichaud L, Charrel RN, Gradoni L. Phlebotominae sandflies and the spreading of leishmaniasis and other diseases of public health concern. Med Vet Entomol 2013;27: 123-47. https://doi.org/10.1111/j.1365-2915.2012.01034.x.

3. Fraga DBM, Pacheco LV, Borja LS, Tuy PGSE, Bastos LA, Solcà MS, et al. The rapid test based on Leishmania infantum chimeric rK28 protein improves the diagnosis of canine visceral leishmaniasis by reducing the detection of false-positive dogs. PLoS Negl Trop Dis 2016;10:e0004333. https://doi.org/10.1371/journal. pntd0004333.

4. Hosein S, Blake DP, Solano-Gallego L. Insights on adaptive and innate immunity in canine leishmaniosis. Parasitology 2017;144: 95-115. https://doi.org/10.1017/S003118201600055X.

5. Grimaldi Jr G, Teva A, Ferreira AL, dos Santos CB, de-Souza Pinto I, de-Azevedo CT, et al. Evaluation of a novel chromatographic immunoassay based on dual-path platform technology (DPP ${ }^{\circledR}$ CVL Rapid Test) for the serodiagnosis of canine visceral leishmaniasis. Trans R Soc Trop Med Hyg 2012;106:54-9. https://doi.org/10.1016/ j.trstmh.2011.10.001.

6. Courtenay O, Carson C, Calvo-Bado L, Garcez LM, Quinnell RJ. Heterogeneities in Leishmania infantum infection: using skin parasite burdens to identify highly infectious dogs. PLoS Negl Trop Dis. 2014 Jan;8:e2583. https://doi.org/10.1371/journal.pntd.0002583.
7. Figueiredo FB, Madeira MF, Nascimento LD, Abrantes TR, MoutaConfort E, Passos SRL, et al. Canine visceral leishmaniasis: study of methods for the detection of IgG in serum and eluate samples. Rev Inst Med Trop São Paulo 2010;52(4):193-6. https://doi.org/10. 1590/S0036-46652010000400005.

8. Tafuri WL, de Oliveira MR, Melo MN, Tafuri WL. Canine visceral leishmaniosis: a remarkable histopathological picture of one case reported from Brazil. Vet Parasitol. 2001;96(3):203-12. https://doi. org/10.1016/s0304-4017(00)00436-2.

9. Paltrinieri S, Gradoni L, Roura X, Zatelli A, Zini E. Laboratory tests for diagnosing and monitoring canine leishmaniasis. Vet Clin Pathol 2016;45:552-78. https://doi.org/10.1111/vcp.12413.

10. Farahmand M, Nahrevanian H. Application of recombinant proteins for serodiagnosis of visceral leishmaniasis in humans and dogs. Iran Biomed J 2016;20:128-34. https://doi.org/10.7508/ibj. 2016.03.001.

11. Sato CM, Sanchez MCA, Celeste BJ, Duthie MS, Guderian J, Reed $\mathrm{SG}$, et al. Use of recombinant antigens for sensitive serodiagnosis of american tegumentary leishmaniasis caused by different Leishmania species. J Clin Microbiol 2017;55:495-503. https://doi.org/ 10.1128/JCM.01904-16.

12. Abass E, Bollig N, Reinhard K, Camara B, Mansour D, Visekruna A, et al. rKLO8, a novel Leishmania donovani - derived recombinant immunodominant protein for sensitive detection of visceral leishmaniasis in Sudan. PLoS Negl Trop Dis. 2013 July 18;7:e2322. https://doi.org/10.1371/journal.pntd.0002322.

13. Quinnell RJ, Courtenay O, Davidson S, Garcez L, Lambson B, Ramos P, et al. Detection of Leishmania infantum by PCR, serology and cellular immune response in a cohort study of brazilian dogs. Parasitology 2001;122:253-61. https://doi.org/10.1017/ s0031182001007363.

14. Abad LPM, Almeida CS, Mattos AMM, Mendonça ACP, Alves MJM, Pinheiro AC, et al. Diagnostic accuracy of rKLO8 versus rK26 ELISAs for screening of canine visceral leishmaniasis. Acta Trop 2016;166:133-8. https://doi.org/10.1016/j.actatropica.2016.11. 021.

15. Badaró R, Benson D, Eulálio MC, Freire M, Cunha S, Netto EM, et al. rK39: a cloned antigen of Leishmania chagasi that predicts active visceral leishmaniasis. J Infec Dis 1996;173:758-61. https:// doi.org/10.1093/infdis/173.3.758.

16. Porrozzi R, da Costa MVS, Teva A, Falqueto A, Ferreira AL, dos Santos CD, et al. Comparative evaluation of enzyme-linked immunosorbent assays based on crude and recombinant leishmanial antigens for serodiagnosis of symptomatic and asymptomatic Leishmania infantum visceral infections in dogs. Clin Vaccine Immunol. 2007;14:544-8. https://doi.org/10.1128/CVI.00420-06.

17. Dantas-Torres F, Miró G, Bowman DD, Gradoni L, Otranto D. Culling dogs for zoonotic visceral leishmaniasis control: the wind of change. Trends Parasitol 2019;35:97-101. https://doi.org/10.1016/j. pt.2018.11.005.

18. Sevá AP, Ovallos FG, Amaku M, Carrillo E, Moreno J, Galati EAB, et al. Canine-based strategies for prevention and control of leishmaniasis in Brazil. PloS One 2016 July 29;11:e0160058. https://doi. org/10.1371/journal.pone.0160058.

19. Peixoto HM, de Oliveira MRF, Romero GAS. Serological diagnosis of canine visceral leishmaniasis in Brazil: systematic review and meta-analysis. Trop Med Int Health 2015;20:334-52. https://doi. org/10.1111/tmi.12429. 
20. da Silva DA, Madeira MF, Abrantes TR, Barbosa Filho CJL, Figueiredo FB. Assessment of serological tests for the diagnosis of canine visceral leishmaniasis. Vet J 2013;195:252-3. https://doi.org/ 10.1016/j.tvjl.2012.06.010.

21. Fernandes CB, Magalhães Junior JT, de Jesus C, Souza BMPS, Laranjeira DF, Fraga DBM, et al. Comparison of two commercial vaccines against visceral leishmaniasis in dogs from endemic areas: IgG, and subclasses, parasitism, and parasite transmission by xenodiagnosis. Vaccine 2014;32:1287-95. https://doi.org/10.1016/j. vaccine.2013.12.046.

22. Lauricella MA, Maidana CG, Frias VF, Romagosa CM, Negri V, Benedetti R, et al. An rK28-based immunoenzymatic assay for the diagnosis of canine visceral leishmaniasis in Latin America. Am J Trop Med Hyg 2016;95:92-8. https://doi.org/10.4269/ajtmh.130768.

23. Dantas-Torres F, Sales KGS, da Silva LG, Otranto D, Figueredo LA. Level of agreement between two commercially available rapid serological tests and the official screening test used to detect Leishmania seropositive dogs in Brazil. Vet J 2018;234:102-4. https://doi.org/10.1016/j.tvjl.2018.02.007.

24. Venturin GL, Bragato JP, Silva KLO, de Lima VMF. Recombinant K28 antigen in ELISA in the diagnosis of canine visceral leishmaniosis. Parasite Immunol 2015;37:670-3. https://doi.org/10.1111/ pim.12281.

25. Quinnell RJ, Carson C, Reithinger R, Garcez LM, Courtenay O. Evaluation of rK39 rapid diagnostic tests for canine visceral leishmaniasis: longitudinal study and meta-analysis. PLoS Negl Trop Dis 2013;7:e1992. https://doi.org/10.1371/journal.pntd. 0001992.
26. Boarino A, Scalone A, Gradoni L, Ferroglio E, Vitale F, Zanatta R, et al. Development of recombinant chimeric antigen expressing immunodominant B epitopes of Leishmania infantum for serodiagnosis of visceral leishmaniasis. Clin Diagn Lab Immunol 2005;12: 647-53. https://doi.org/10.1128/CDLI.12.5.647-653.2005.

27. Grimaldi Jr G, Teva A, dos-Santos CB, Santos FN, Pinto IS, Fux B, et al. Field trial of efficacy of the Leish-tec ${ }^{\circledR}$ vaccine against canine leishmaniasis caused by Leishmania infantum in an endemic area with high transmission rates. PLoS One 2017;12(9):e0185438. https://doi.org/10.1371/journal.pone.0185438.

28. Testasicca MCS, dos Santos MS, Machado LM, Sefuro AV, Doro D, Avelar D, et al. Antibodies responses induced by Leish-Tec ${ }^{\mathbb{B}}$, an A2-based vaccine for visceral leishmaniasis, in a heterogeneous canine population. Vet Parasitol 2014;204:169-76. https://doi.org/ 10.1016/j.vetpar.2014.04.025.

29. Souza NA, Leite RS, Silva SO, Penna MG, Vilela LFF, Melo MN, et al. Detection of mixed Leishmania infections in dogs from an endemic area in southeastern Brazil. Acta Trop 2019;193:12-7. https://doi.org/10.1016/j.actatropica.2019.02.016.

30. Vale AM, Fujiwara RT, da Silva Neto AF, Miret JA, Alvarez DCC, da Silva JCF, et al. Identification of highly specific and cross-reactive antigens of Leishmania species by antibodies from Leishmania (Leishmania) chagasi naturally infected dogs. Zoonoses Public Health 2009;56:41-8. https://doi.org/10.1111/j.1863-2378.2008. 01183.x.

31. Paz GF, Rugani JMN, Marcelino AP, Gontijo CMF. Implications of the use of serological and molecular methods to detect infection by Leishmania spp. in urban pet dogs. Acta Trop 2018;182:198-201. https://doi.org/10.1016/j.actatropica.2018.03.018. 